\title{
Network Mode based on Multi-communication Mechanism
}

\author{
Fan Yibin, Liu Zhifeng, Zhang Sheng, Lin Ying
}

Department of Military Finance, Military Economy Academy, Wuhan City Hubei Province ZipCode 430035, China

fanyibin_wh@163.com

Keywords: Cognitive Radio, Energy-saving, PSO (Particle Swarm Optimization), Annealing algorithm

Abstract. For the sake of improving green energy-saving efficiency of the radio communication system,we proposed a green energy-saving algorithm for multichannel communication system based on the energy consumption model analysis. Firstly, mathematical model of the multi-channel energy consumption minimization problem was built. Secondly, we used PSO algorithm to solve mathematical model, and annealing operation of simulated annealing algorithm was introduced into the PSO algorithm, which would prevent the local optimal solution and promote the speed of solving. In the end, we used the simulation contrast experiments to test our algorithm performance. The results show that our algorithm can meet different user service requirements, and can reduce total transmission power and energy consumption of the system, which is in favor of environment protection.

\section{Introduction}

Along with the popularization and mature of computer and moving communication technology, mobile user data increase sharply, which brings soaring energy consumption and environment problems. Energy consumption has attracted extensive attention, and the energy related economic cost has reduced the telecom profits. Therefore, we proposed the green communication technology to save energy. Green communication technology not only reduce emission of $\mathrm{CO}_{2}$ and disadvantageous effect on environment, but also reduce telecom economic cost, which has become the current research highlights all over the world ${ }^{[1][2]}$.

For the sake of system power reduction and improving energy utilization, we proposed a new green energy-saving algorithm for multichannel communication system. Firstly, we built a mathematical model for multichannel power consumption minimization problem. Secondly, we used combinational algorithm of SA(simulated annealing algorithm) and PSO (particle swarm optimization algorithm)to solve this model. In the end, we used the simulation contrast experiments to test our algorithm performance.

\section{Power Consumption Minimization Problem of Multichannel}

\subsection{System Model}

Assuming that all channels are free and can be used by cognitive radio system, the power consumption minimization problem of multichannel can be described as following:

$$
\begin{aligned}
& \min _{\left\{P_{n}\right\}} \sum_{n=1}^{N} \hat{P}_{n} \\
& \text { s.t. } \\
& \sum_{n=1}^{N} b_{n}=b
\end{aligned}
$$

Where, $n$ is the number of channels, $b$ is target speed, $p_{n}$ is transmission power of branch $n$,

$\hat{P}_{n}$ is system energy consumption, and $b_{n}$ is real speed of branch $n$. 


\subsection{Energy consumption model}

The system energy consumption of branch $\mathrm{n}$ also can be modeled as following:

$$
\hat{P}_{n}=\frac{P_{n}}{\bar{\eta}_{n}}
$$

Where, $\bar{\eta}_{n}$ is the average efficiency of PA in branch $n$.

The approximate model of average PA efficiency is :

$$
\bar{\eta}=\eta(P)
$$

Therefore, the energy consumption expression of channel npower amplifying system is:

$$
\hat{P}_{n}=\frac{P_{n \cdot \max }^{\alpha_{n}}}{\eta_{\max . n}} \cdot P_{n}^{1-\alpha_{n}}
$$

Informed by expression (4), the power consumption of the system depends on active channel number. And we can convert the problems into transmission power minimum value problem, under the condition of satisfying target certain rate limit, and under the condition of system power minimum consumption ${ }^{[12]}$.Supposing in the multi-channel and SNR(signal to noise ratio) known conditions, we choose $\mathrm{N}$ channels of these as active channels to transmit power, and at this moment, total power consumption of the system is as following:

$$
\hat{P}_{n}=N \cdot \frac{P_{n \cdot \max }}{\eta_{\text {max.A }}}
$$

Where, $\mathrm{N}$ is the number of active channels. As a result, we list the Lagrange equation about PA minimum transmission power:

$$
\begin{aligned}
f= & \sum_{n=1}^{N} P_{n}+\lambda\left(b-\sum_{n=1}^{N} b_{n}\right) \\
& =\sum_{n=1}^{N} P_{n}+\lambda\left(b-\frac{1}{\ln 2} \sum_{n=1}^{N} \ln \left(1+P_{n} g_{n}\right)\right)
\end{aligned}
$$

In equation (6), we get $P_{n}$ and $\lambda$ partial derivatives as following:

$$
\begin{aligned}
& \frac{\partial f}{\partial P_{n}}=1-\frac{\lambda}{\ln 2} \frac{1}{g_{n}^{-1}+P_{n}}=0 \\
& \frac{\partial f}{\partial \lambda}=\frac{1}{\ln 2} \sum_{n=1}^{N} \ln \left(1+P_{n} g_{n}\right)-b=0
\end{aligned}
$$

For the equation (1), we used combinational algorithm of SA (simulated annealing algorithm) and PSO (particle swarm optimization algorithm) to solve it.

\section{Simulated Annealing Particle Swarm Optimization Algorithm(SA-PSO)}

\section{1 particle swarm algorithm}

In the PSO, each initialized particle represents the solution of optimization problem, and each particle can be evaluated its quality by fitness function in each iteration process. And in the searching process, each individual particle's optimal solution and whole population's global optimal solution are updated constantly. In the next iteration, each particle's speed and position are updated by tracing two extreme values in the last iteration. Accordingly, we get the global optimal solution by constant iteration. The equation of speed and position are as following:

$$
\left\{\begin{array}{l}
V_{i d}^{k+1}=\omega V_{i d}^{k}+c_{1} r_{1}\left(P_{i d}^{k}-X_{i d}^{k}\right)+c_{2} r_{2}\left(P_{g d}^{k}-X_{i d}^{k}\right) \\
X_{i d}^{k+1}=X_{i d}^{k}+V_{i d}^{k+1}
\end{array}\right.
$$

Where, $\mathrm{d}$ is the dimensions of solution space, $\mathrm{i}$ is the particle number in the population, $\omega$ is the inertia weight, $\mathrm{k}$ is current iteration number, $\mathrm{c} 1, \mathrm{c} 2$ are the acceleration factor, $\mathrm{r} 1, \mathrm{r} 2$ are the random number in $[0,1]$ interval. 


\section{2 simulated annealing algorithm}

simulated annealing'algorithm is a kind of heuristic algorithm with high partial search ability, whose simulated solid annealing process is suitable for solving large scale combinatorial optimization problem, and uses Metropolis criteria to accept probability $\mathrm{P}$ of the optimal solution, as following:

$$
P= \begin{cases}1 & f(i) \leq f\left(i^{\prime}\right) \\ \exp \left(\frac{f(i)-f\left(i^{\prime}\right)}{t}\right) & f(i)>f\left(i^{\prime}\right)\end{cases}
$$

Where, $f(i)$ is the objective function of the problems, $t$ is controls parameter ${ }^{[14]}$.

Supposing that $\mathrm{T}(t)$ represents temperature at $t$ moment, then SA simulates cooling way as following:

$$
T(t)=\frac{T_{0}}{\lg (1+t)}
$$

Sphere function has only one global optimal value; Griewank function is not smooth and continuous in the near global optimal value; Rastrigin is pathological function hardly finding the global optimal value; the running results of PSO and SA-PSO is as shown in figure 2. Informed by figure 1, the convergence rate of SA-PSO is much better than comparing algorithm in all functions, which indicates SA-PSO has much better global search ability, convergence accuracy and rate.

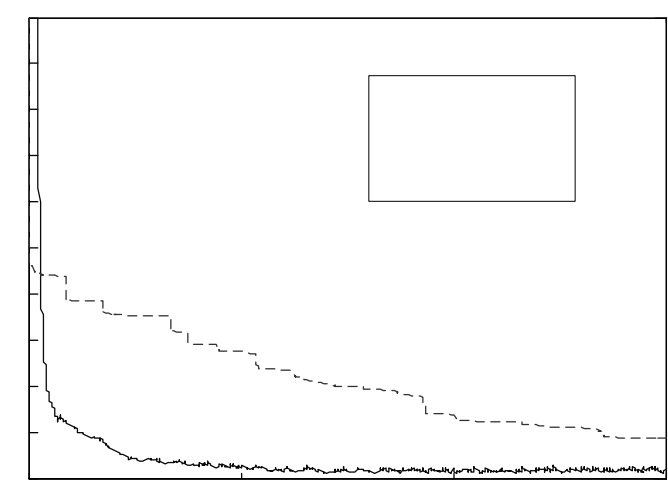

(a) Sphere function fitness convergence curve

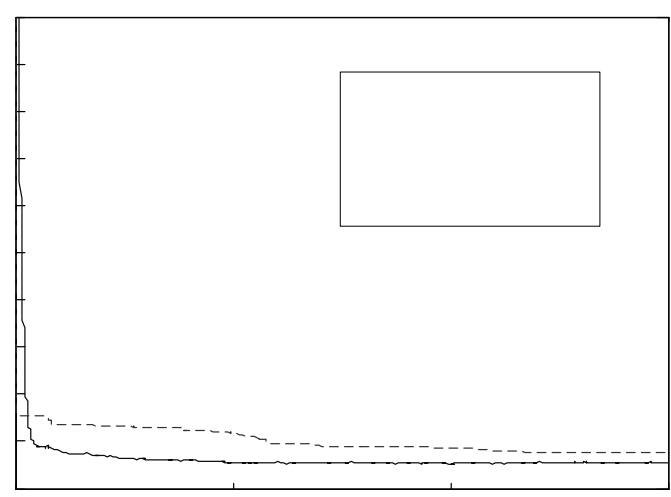

(b) Griewank function fitness evolution curve 


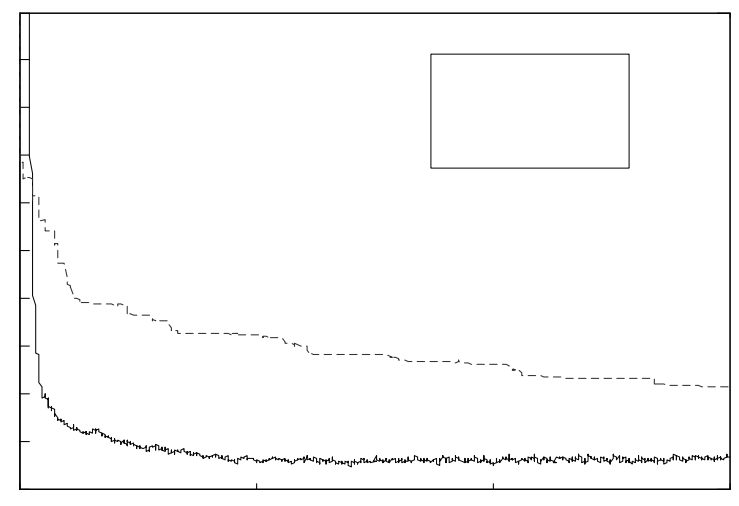

(c) Rastrigin function fitness evolution curve

Figure 1 performance comparison between SA-PSO and PSO

\section{Simulation Environment}

In order to test the green energy-saving performance of SA-PSO, we choose Matlab 2012 to make simulation experiment under the condition of Windows XP with P4 double cores CPU and 4GRAM. At the same time, we carry on comparison experiment between SA and PSO to make this article more convincing.

\subsection{Energy utilization comparison}

For 3 channels communication system, energy utilization of several algorithm is as shown in figure 2. Informed by figure 2, compared with the single SA or PSO, the average energy utilization of SA-PSO is the highest, and the energy-saving efficiency is greatly improved up to $75.02 \%$, which is far more than $61.33 \%$ of SA or $66.08 \%$ of PSO. This mainly because SA-PSO integrates GA (genetic algorithm) strong global search ability and SA strong local search ability. This algorithm can overcome the local optimal solution and choose maximum SNR channel to use, and can minimize transmission power, which could achieve the energy-saving effect.

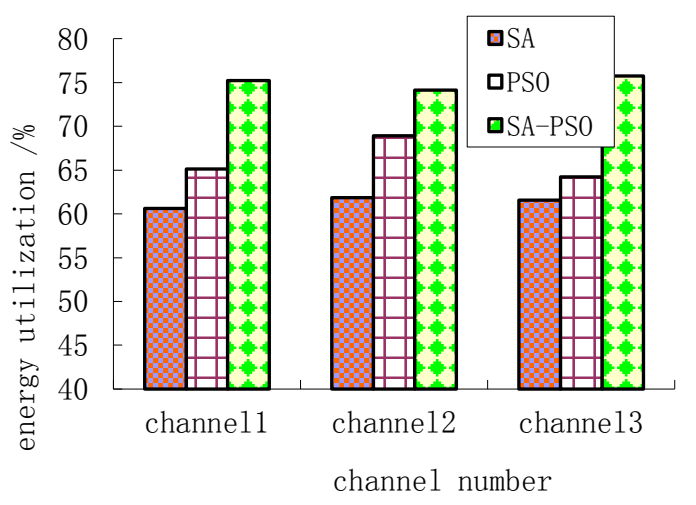

Figure 2 the energy utilization contrast by different algorithms

\section{2 performance analysis of different channels}

In order to further test SA-PSO universality, we take three different user service requirements into account, as shown in table 1 . Pattern 1 is used in video communication, and pattern 2 is used in voice communication, and pattern 3 is used in LDR (low data rate).

Table 1 description of different patterns

\begin{tabular}{cccc}
\hline & pattern 1 & pattern 2 & pattern 3 \\
\hline BER $_{\text {tar }}$ & $10^{-3}$ & $10^{-2}$ & $10^{-6}$ \\
DER $_{\text {tar }}$ & $300 \mathrm{kbps}$ & $10 \mathrm{kbps}$ & $200 \mathrm{kbps}$ \\
\hline
\end{tabular}


The maximum bit error rate, data rate and total transmission power of different algorithms for different patterns are shown in figure2. Informed by figure 2, in the condition of meeting different customers service request, total transmission power and maximum bit error rate of SA-PSO is minimum, and the data rate is maximum. The results indicate that SA-PSO can efficiently reduce energy consumption, and is more in favor of environment protection.

\section{Conclusions}

Green, cognitive radio is a kind of green communication technology. In order to improve energy utilization and reduce energy consumption, we propose a green energy-saving algorithm based on the energy consumption model analysis, and make performance analysis by simulation experiment. Simulation experiment indicates that compared with other algorithms, our algorithm not only reduce total transmission power of the system, but also better meet customer service requirements for quality.

\section{References}

[1]Lv, Zhihan, Liangbing Feng, Haibo Li, and Shengzhong Feng. "Hand-free motion interaction on Google Glass." In SIGGRAPH Asia 2014 Mobile Graphics and Interactive Applications, p. 21. ACM, 2014.

[2]Li, Wubin, Johan Tordsson, and Erik Elmroth. "An aspect-oriented approach to consistency-preserving caching and compression of web service response messages." In Web Services (ICWS), 2010 IEEE International Conference on, pp. 526-533. IEEE, 2010.

[3]Lv, Zhihan, and Tianyun Su. "3D seabed modeling and visualization on ubiquitous context." In SIGGRAPH Asia 2014 Posters, p. 33. ACM, 2014.

[4]Lv, Zhihan, Liangbing Feng, Shengzhong Feng, and Haibo Li. "Extending Touch-less Interaction on Vision Based Wearable Device." Virtual Reality (VR), 2015 iEEE. IEEE, 2015.

[5]Zhang, Mengxin, Zhihan Lv, Xiaolei Zhang, Ge Chen, and Ke Zhang. "Research and Application of the 3D Virtual Community Based on WEBVR and RIA." Computer and Information Science 2, no. 1 (2009): p84. 